\title{
Simulated microgravity: An efficient model for the study of age-related bone and muscle diseases
}

\author{
Umberto Tarantino ${ }^{1,2}$, Ida Cariati ${ }^{1,3 *}$, Virginia Tancredi ${ }^{4,5}$, Mario Marini ${ }^{4,5}$, Donato Casamassima ${ }^{2}$, Francesco Romano ${ }^{2}$ Riccardo Iundusi $^{2}$ \\ and Elena Gasbarra ${ }^{2}$ \\ ${ }^{1}$ Department of Clinical Sciences and Translational Medicine, "Tor Vergata" University of Rome, Via Montpellier 1, 00133 Rome, Italy \\ ${ }^{2}$ Department of Orthopaedics and Traumatology, "Policlinico Tor Vergata" Foundation, Viale Oxford 1, 00133 Rome, Italy \\ ${ }^{3} \mathrm{PhD}$ Students' Program in Medical-Surgical Biotechnologies and Translational Medicine, "Tor Vergata” University of Rome, Via Montpellier 1, 00133 Rome, Italy \\ ${ }^{4}$ Department of Systems Medicine, "Tor Vergata” University of Rome, Via Montpellier 1, 00133 Rome, Italy \\ ${ }^{5}$ Center of Space BioMedicine, "Tor Vergata" University of Rome, Via Montpellier 1, 00133 Rome, Italy
}

\begin{abstract}
Spaceflights short and long duration are known to induce a series of physiological and environmental stresses, with a consequent impact on normal biological processes, such as the functional integrity of muscles and bone mass. Studies on animal and human models indicate that muscle loss can occur rapidly with disuse or immobilization, and that muscle atrophy contributes to bone loss mainly through increased osteoclastogenesis. In addition, since many of changes observed in astronauts during spaceflight recall the onset and progression of typical ageing diseases (such as osteoporosis, arthrosis, and sarcopenia), the musculoskeletal system has been a major focus of simulated microgravity research over the last decade. Based on this evidence, in this review we have examined the effects of simulated microgravity on muscle-bone crosstalk, to clarify the cellular and molecular mechanisms that cause muscle and bone loss following alteration of normal mechanical load. The understanding of these mechanisms is fundamental for the development of therapeutic approaches aimed at inhibiting muscle and bone loss and, therefore, preventing bone fractures.
\end{abstract}

\section{Introduction}

Fragility fractures in older adults are often preceded by a loss of muscle mass and strength. Similarly, bone loss that occurs with prolonged bed rest or exposure to simulated microgravity is also preceded by rapid loss of muscle mass [1].

It is known that the hostile environment of space has a significant impact on human physiology. Specifically, exposure to simulated microgravity causes a substantial mechanical unloading of mammalian tissues, resulting in rapid alterations of their physiology, which represents a significant risk for the long period of human stay in space [2-4].

Many of the pathophysiological adaptations that occur during spaceflight cannot be adequately counteracted by exercise or nutritional supplementation. Therefore, in order to develop highly effective countermeasures and prevent spaceflight induced diseases, it is essential to discover the underlying molecular mechanisms, so as to also understand the pathophysiology of diseases occurring on Earth, such as osteoporosis, muscle atrophy, cardiovascular diseases and immune system dysfunctions.

Based on this evidence, the aim of our review was to summarize the current scientific knowledge on the cellular and molecular mechanisms that are at the basis of muscle-bone crosstalk and that occur under simulated microgravity conditions, so as to promote the development of therapeutic approaches to inhibit the loss of bone and muscle mass and, at the same time, prevent the onset of fragility fractures.

\section{Simulated microgravity}

The absence of gravity in space, also known as zero gravity, can be simulated on Earth as microgravity. Simulated microgravity is a condition in which a system is subject to a gravitational field of very low value and is studied in different scientific and technological fields to highlight phenomena that on Earth are masked by the effects of the high gravitational field.

It is known that the gravitational field played an important role in shaping evolution when life moved from water to land, thus influencing much of the physical and biological phenomena throughout Earth's history [5]. Initially it was believed that gravity influenced natural selection only by limiting the range of acceptable body size; according to this principle, living organisms should develop systems to provide cell membrane rigidity, fluid flow regulation and appropriate structural support for locomotion [5]. The first empirical experiments, mostly conducted by Russian scientists in the 1960s, were unable to

${ }^{\star}$ Correspondence to: Ida Cariati, $\mathrm{PhD}$ Students' Program in Medical-Surgical Biotechnologies and Translational Medicine, Department of Clinical Sciences and Translational Medicine, “Tor Vergata” University of Rome, Via Montpellier 1,00133 Rome, Italy, E-mail: ida.cariati@uniroma2.it

Key words: aging, fragility fractures, muscle-bone crosstalk, osteoporosis, sarcopenia, simulated microgravity

Received: October 15, 2020; Accepted: October 28, 2020; Published: November 04,2020 
demonstrate the existence of profound changes caused by exposure to simulated microgravity, but instead fed false notions that the near absence of gravity has no significant effect on living organisms [6,7].

Only later, with the beginning of the first investigations in the space environment, it was discovered that biological properties change with the decrease of gravitational force, emphasizing the relationship between physical force and biological function [5]. In fact, it has been observed that cells exposed to simulated microgravity can be deeply affected by the physical changes that occur in this unique environment and that include the loss of gravity-dependent convection, negligible hydrodynamic shear and lack of sedimentation $[8,9]$. These changes can significantly alter cell responses to a wide array of environmental and internal biophysical stresses [10]. As a result, enzymatic, genetic, and epigenetic processes change, causing modifications in cells and tissues shape, function, and behavior $[11,12]$.

The main damages caused by the gravitational environment include loss of bone [13-15] and muscle mass [16,17], cardiovascular dysfunction $[18,19]$, alteration in the fracture healing processes $[20,21]$ and wounds repair [22], impairment of the immune response [23,24]. Therefore, since it is known that simulated microgravity can disrupt fundamental mechanisms of different biological processes, these studies are used both to investigate the effects of permanence in space on astronauts and to identify mechanisms involved in "terrestrial" pathologies.

The musculoskeletal system: The musculoskeletal system, an organ system comprised primarily of the skeleton and skeletal muscle, has been one of the main focuses of simulated microgravity research in the last ten years. These studies have focused on the evidence that many of the changes observed in astronauts during spaceflights are reminiscent of the onset and progression of diseases associated with aging on Earth, such as osteoporosis, arthrosis, and sarcopenia.

The skeleton system: Bone is a multifunctional tissue that performs two main functions: to provide mechanical integrity for both movement and protection, and to help regulate mineral homeostasis through involvement in metabolic pathways [25].

It is known that biomechanical forces play a critical role in the development of the skeletal system and that the architecture of bone is related to the mechanical stresses that are exerted on it [26-30]. The skeleton, in fact, is a metabolically active organ that undergoes continuous remodeling throughout life, during which mature bone tissue is removed from the skeleton (a process called bone resorption) and new bone tissue is formed (a process called ossification) [31].

Two main types of cells are responsible for bone metabolism: osteoblasts, which secrete new bone, and osteoclasts, which break down bone. To maintain bone homeostasis, it is essential that there is close collaboration between these two types of cells and other cell populations present in bone remodeling sites, such as immune cells [32].

An imbalance in the regulation of the two processes of bone remodeling promotes the onset of many metabolic bone diseases, including osteoporosis [33].

Physiological effects of simulated microgravity on bone cells: In a simulated microgravity environment, mechanical stress is non-existent, resulting in rapid and severe decoupling between bone formation and bone resorption.

It is known that simulated microgravity causes immense changes in bone tissue that result in a decrease in bone mass and lead to early onset osteoporosis in many astronauts [34-37]. These changes produce a condition known as spaceflight osteopenia, which is characterized by an increase in bone resorption by osteoclasts and a decrease in bone formation by osteoblasts [36].

In recent years, many studies have been conducted to identify the alterations and mechanisms induced by simulated microgravity at the cellular level, with particular attention to the effects of simulated microgravity on mesenchymal stem cells, osteoblasts, and osteoclasts.

Mesenchymal Stem Cells: Mesenchymal Stem Cells (MSCs) are multipotent stem cells found in the bone marrow and are important for their ability to differentiate into cartilage cells (chondrocytes), bone cells (osteoblasts) and fat cells (adipocytes) [38]. It has been reported that simulated microgravity can induce physiological changes in MSCs, including maintaining them in an undifferentiated state and enhancing cell proliferation and differentiation in a single lineage [39]. For example, it has been observed that loss of gravity in space or lack of physical activity due to spinal and brain injury can significantly reduce mechanical stress, leading to a decreased rate of osteogenesis and an increased rate of adipogenesis [40,41].

The lack of mechanical stimuli seems to keep MSCs in a quiescence state; however, the exact mechanism of simulated microgravity induced osteogenic-adipogenic shift is not yet clear. One of the most reliable hypotheses is that the simulated microgravity acts on MSCs by suppressing osteogenesis through the inhibition of the MitogenActivated Protein Kinase (MAPK) signaling pathway and promoting adipogenesis by increasing Peroxisome Proliferator-Activated Receptor gamma (PPAR- $\gamma$ ) expression [42]. Alternatively, the inhibition of osteogenic differentiation of MSCs and osteogenic precursor cells induced by simulated microgravity could be attributed to F-actin cytoskeletal disorders [43] and alterations of Rho GTPase activity $[41,44,45]$.

Osteoblasts: MSCs can differentiate into osteoblasts through a complex process, regulated by numerous transcription and growth factors [46]. Under normal conditions, the up-regulation of these factors induces an increase in Alkaline Phosphatase (ALP), Osteocalcin (OCN) expression and bone formation; on the contrary, if the factors involved in the differentiation process are altered, bone loss may occur [47].

It is known that osteoblasts are responsible for bone formation and play an important role in maintaining bone homeostasis [48]. Osteoblast dysfunction may be the main cause of bone loss [49]. Carmeliet et al. stated that ALP expression and activity decrease significantly following exposure to simulated microgravity, and other studies have also shown a trend toward a decrease in bone formation markers, including ALP and OCN [50].

It has also been observed that simulated microgravity can inhibit the differentiation of osteo-progenitor cells into mature osteoblasts [34,41], as well as reduce the osteogenic potential of bone marrow MSCs [51]. Based on this evidence, it has been hypothesized that bone loss induced by simulated microgravity could be attributed to osteoblasts, because of their reduced activity and proliferation, reduced differentiation, and decreased reactivity to bone-related factors in the microenvironment [52].

Although these mechanisms have not yet been fully understood, numerous scientific evidences have shown that in vitro osteoblastic cells are sensitive to simulated microgravity [53,54]. Specifically, it has been observed that exposure to simulated microgravity induces alterations 
in the cytoskeletal organization in ROS17/2.8 cells, a mature clonal osteoblastic cell line that responds to stretch by proliferating when exposed to the gravitational environment [55]. There is also growing evidence that the cytoskeleton is closely related to nuclear morphology and function [56], and the enlarged nuclei observed in flight osteoblasts could be a result of cytoskeletal disruption [13]. In addition, it has also been shown that actual or simulated weightlessness disrupts osteoblasts microfilaments, resulting in defective bone formation [57,58], and that the disorganization of microfilaments is caused by the inhibition of Rho by Random Positioning Machine (RPM) [59].

Osteoclasts: Several studies suggest that simulated microgravity leads to a decrease in osteoblast function and an increase in osteoclastogenesis, thus contributing to bone loss [60-62].

It is known that osteoclasts are multinucleated bone-resorbing cells and can degrade mineralized matrices, such as bone and calcified cartilage [63]. It has been suggested that osteoclast differentiation improves under simulated microgravity conditions, since an increase in collagen telopeptides was observed in media samples retrieved from space [64]. Finally, from a molecular point of view, it has been observed that under simulated microgravity conditions, several elevated growth factors in pre-osteoclast cells can alter Receptor Activator of Nuclear Factor Kappa-B Ligand (RANKL) and Osteoprotegerin (OPG) levels in bone environments and thus facilitate increased osteoclast activity [65].

The skeletal muscle: The muscles are necessary for movement and to counteract gravity, and they must be used to maintain structure and function. It is known that muscle mass and strength may decrease in response to actual and simulated microgravity exposure [66]. In fact, the absence of load forces during spaceflight can cause muscle atrophy, changes in muscle fiber composition and gene expression, as well as a reduction in regenerative muscle growth $[17,67]$.

Undoubtedly, skeletal muscle mass loss in response to simulated microgravity exposure has been a medical and physiological concern since the first space missions [68] and, together with changes in neural drive, is one of the main determinants of the decline in muscle strength and power [69]. However, despite the considerable knowledge acquired on the physiological changes induced by spaceflight, the mechanisms of muscle atrophy and the effectiveness of in-flight countermeasures have yet to be fully clarified.

Experiments conducted on animal models have shown that postural muscles, generally containing a higher percentage of slow fibers, are more prone to atrophy than non-postural muscles [70-72]. Similar results have also been found for human skeletal muscles, since the effects of simulated microgravity seem to be more evident in antigravity muscles, that is muscles that play a postural role under standard gravity conditions $(1 \mathrm{~g})$, such as the calf muscles (soleus and gastrocnemius) and the quadriceps [73-75].

In fact, during spaceflight or in a microgravity environment, humans do not need to support their bodies; so, the antigravity muscles become atrophied [66]. It has been observed that after only 8 days of spaceflight, the volume of quadriceps and triceps surae decreased by -6.0 and $-6.3 \%$, respectively, and from -5.5 to $-15.4 \%$ and from -8.8 to $-15.9 \%$, respectively, after $9-16$ days of spaceflight $[76,77]$.

Most studies have also shown that extensor muscles are more affected by simulated microgravity than their antagonistic counterparts, the flexors [75]. For example, Widrick et al. observed that flexor fibers are less affected than extensor fibers following a 17-day spaceflight [78]; however, as the duration of the mission lengthened, all skeletal muscles seemed to be affected by simulated microgravity conditions in a similar way [73].

Existing data on the effect of spaceflight on human skeletal muscles are quite heterogeneous, both in terms of size and rate of atrophy, probably due to various factors, such as age, pre-flight fitness level and nutritional status, as well as content and adherence to on-board countermeasures [67].

Discordant data also concern the type of muscle fiber mainly affected by atrophy in simulated microgravity conditions. Several studies conducted on rats have shown that type I muscle fibers, which are slow and resistant to fatigue, atrophy preferentially in simulated microgravity $[16,75]$. Regarding to human skeletal muscles, in some cases it has been observed that type II fibers were affected as much as type I fibers were $[75,78,79]$; in other cases, however, it has been shown that prolonged exposure to the gravitational environment induced a significant decrease in the cross-sectional area of fibers from the vastus lateralis muscle, with a greater decline in type II fibers and a smaller decline in type I fibers [79].

Muscle-bone crosstalk: Bone and muscle tissue share a common destiny throughout the life of an individual, not only from a functional but also morphological and structural point of view [80].

Initially, it was thought that the muscle-bone crosstalk was only mechanical in nature: bone provides attachment sites for muscle, and skeletal muscle imparts a force on the bone to facilitate the body's locomotion [81]. The bone, in fact, can adjust their mass and structure according to the variations of the mechanical load applied by the muscle. This mechanical perspective implies that a decline in muscle function causes a decrease in the loading of bone, which results in bone loss [82]. However, the reduction in bone mass does not fully explain the occurrence of sarcopenia, nor does muscle atrophy account for the totality of osteoporosis [83], although sarcopenia and osteoporosis often develop in parallel in many patients. In this regard, the term "osteosarcopenia" has recently been coined to indicate the coexistence of these two chronic musculoskeletal conditions that are associated with aging [84]. Osteoporosis, a condition of low bone mass and microarchitectural deterioration bone [85], and sarcopenia, the loss of muscle mass, strength and function [86], often coexist in a fragile subgroup of the elderly population, leading to significantly worse outcomes than those observed in both conditions alone.

The molecular mechanisms that cause both muscle and bone loss are still unclear. However, the scientific literature agrees in supporting the paradigm that the nature of muscle-bone crosstalk is not only mechanical, but also biochemical, since muscle and bone tissues are closely related to each other and, when the aging process begins to occur on one of them, the functionality of the other is also affected [87]. From this point of view, sarcopenia and osteoporosis are two sides of the same coin, that of bone fragility, and occur in parallel in the elderly subject.

Muscle-bone biochemical crosstalk: All cellular elements responsible for the metabolic turnover of bone, including osteoblasts, osteocytes, osteoclasts, chondroblasts and chondrocytes, act under the influence of muscle, emphasizing the key role of this tissue in the definition of bone quality.

Emerging evidence suggests that Bone Morphogenetic Proteins (BMPs) play an important role in both muscle and bone homeostasis [88]. BMPs are molecules belonging to the Transforming Growth Factor-Beta (TGF- $\beta$ ) family, which is involved in various biological 
processes related to proliferation, differentiation, morphogenesis, homeostasis, and cell regeneration $[89,90]$. These proteins regulate gene expression by phosphorylation of some transcriptional factors (Smad 1, 5 and 8), while their activity is inhibited by the secretion of factors such as Noggin and Chordin, which prevent the binding of growth factors to their receptor [91].

It has recently been shown that BMP expression plays a role in the control of adult skeletal muscle mass and regeneration [89,92,93], since an association between BMP- 2 and BMP- 4 expression and satellite cell activity has been found [94].

It is known that satellite cells play an indispensable role in muscle regeneration: in fact, the self-renewing proliferation of these cells not only contributes to the maintenance of the stem cell population, but also provides numerous myogenic cells, which proliferate, differentiate, merge and induce the formation of new myofibers, thus promoting the reconstitution of a functional contractile apparatus [95]. Not surprisingly, the loss of satellite cells and/or their degeneration could be related to the altered muscle metabolism that characterizes osteoporotic patients.

It has also been hypothesized that the quality of muscle tissue depends on the balance between BMP signalling and myostatin signalling [96], which is known to affect muscle mass through negative regulation of myogenesis [97]. In vitro experiments have shown that myostatin can block the proliferation of myoblasts, as well as the proliferation and renewal of satellite cells [98].

Based on this evidence, spaceflight and prolonged bed rest can be considered useful human models to understand the effects of mechanical unloading on muscles and bones. In fact, the definition of the mechanisms linking muscle and bone in the context of disuse is fundamental for the development of therapeutic approaches to inhibit muscle and bone mass and ultimately prevent fragility fractures.

Effects of simulated microgravity on muscle-bone crosstalk: Many of changes observed in astronauts during spaceflight recall the onset and progression of diseases associated with aging on Earth, although they occur much faster. Therefore, it has been hypothesized that changes observed in response to simulated microgravity may share the common physiological basis with some of physiological adaptations typical of aging, such as the loss of bone and muscle mass that characterizes the main musculoskeletal disorders [99].

Considering that primary cultures of human satellite cells represent a useful scientific model to identify biomolecular processes involved in muscle mass loss linked to the alteration of the normal mechanical load, in a recent study we investigated the role of myostatin and BMP-2 in the response of human satellite cells of control (CTRL), osteoporotic (OP) and osteoarthritic (OA) patients to normogravity and simulated microgravity regimens [99].

Our results showed that in the very early phases of simulated microgravity condition, satellite cells are more active than those subjected to the normogravity regime, as demonstrated by both the increase in the number of myotubes and the significant increase in BMP-2 expression in all experimental groups. However, with prolongated exposure to simulated microgravity regime, satellite cells and new formed myotubes underwent to degeneration and cell death.

We observed that simulated microgravity was also able to induce significant changes in myostatin expression, with group-dependent variations, since OP patients had a significant reduction in myostatin expression compared to CTRL and OA patients [99].

Based on the obtained results, we hypothesized that the main cause of reduced satellite cells function could be alteration of systemic factors, such as BMPs and myostatin, which play important roles in the regulation of muscle growth and atrophy. It is possible that exposure to simulated microgravity amplifies these mechanisms, inducing significant reductions in the size, volume, and strength of skeletal muscles, leading to further muscle atrophy and weakening, as well as subsequent bone loss $[100,101]$. Undoubtedly, this negative influence of simulated microgravity on muscle-bone crosstalk could favor the onset of osteosarcopenia and, therefore, contribute to the development of greater bone fragility (Figure 1).

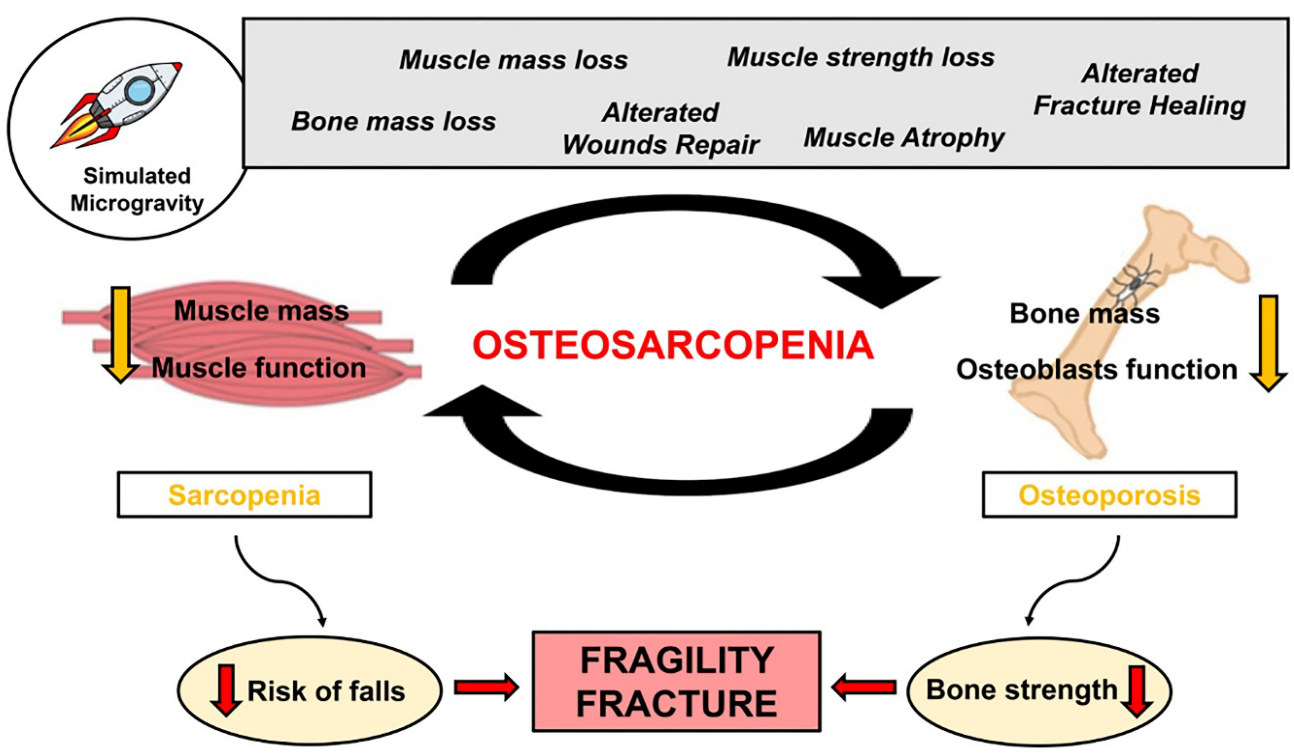

Figure 1. Effects of simulated microgravity on the musculoskeletal system and the pathophysiology of osteosarcopenia 
Bone loss in space is mainly attributable to an imbalance in the bone remodeling process, reflecting increased bone resorption and reduced bone formation [102].

Not surprisingly, it has been observed that astronauts undergo bone mass loss at a rate of 0.5 to $1.5 \%$ per month during a 4 - to 6 -month spaceflight, and that the Bone Mineral Density (BMD) of the proximal femur can only be partially recovered at 1 year after re-exposure to Earth gravity $[103,104]$. It has been proposed that changes in bone and muscle mass under these conditions occur in such a way that muscle atrophy precedes the decline in bone mass $[105,106]$; however, it would appear that muscle loss can be recovered about six times faster than bone loss in astronauts after return to normal gravity [107]. These results suggest that decreased muscle-derived forces may primarily drive bone loss with unloading.

\section{Conclusions}

In this review, we discussed and summarized the effects of spaceflight on living organisms, focusing our attention on the specific effects of simulated microgravity on bone and muscle cells.

Studies on animal and human models indicate that muscle loss can occur rapidly with disuse or immobilization. In addition, muscle atrophy appears to contribute directly to bone loss mainly through increased bone resorption by osteoclasts.

Numerous scientific evidence has suggested significant differences in the proteins and molecules expression involved in muscle-bone crosstalk under simulated microgravity conditions compared to normal gravity; this information is essential to understand the cellular and molecular bases of the main musculoskeletal disorders associated with aging on Earth, with onset and progression similar to many of the changes occurring in astronauts during spaceflight.

The highly integrated changes in skeletal muscle and bone observed in several clinical studies suggest that independent therapeutic approaches targeting sarcopenia or osteoporosis alone may not be sufficient for effective prevention of fragility fractures, and that new strategies are needed to improve both tissues simultaneously. Further studies will be essential to fully characterize the processes involved in the cellular response to simulated microgravity and to identify specific therapeutic targets for the prevention and treatment of bone and muscle pathological conditions related to the alteration of normal mechanical load.

\section{Funding}

This research received no external funding.

\section{Conflicts of interest}

The authors declare that they have no competing interest.

\section{References}

1. Bettis T, Kim BJ, Hamrick MW (2018) Impact of muscle atrophy on bone metabolism and bone strength: implications for muscle-bone crosstalk with aging and disuse. Osteoporos Int 29: 1713-1720. [Crossref]

2. Buckey Jr JC (1999) Preparing for Mars: the physiologic and medical challenges. Eur J Med Res 4: 353-356.

3. Williams DR (2002) Bioastronautics: optimizing human performance through research and medical innovations. Nutrition 18: 794-796.

4. Kalb R, Solomon D (2007) Space exploration, Mars, and the nervous system. Arch Neurol 64: 485-490.
5. Bizzarri M, Monici M, van Loon JJWA (2015) How Microgravity affects the biology of living systems. Biomed Res Int 2015: 863075. [Crossref]

6. Montgomery Jr PO, Cook JE, Reynolds RC, Paul JS, Hayflick L, et al. (1978) The response of single human cells to zero gravity. In Vitro 14: 165-173.

7. Tairbekov MG, Parfyonov GP, EYa S, Sushkov FV (1983) Experimental and theoretical analysis of the influence of gravity at the cellular level: a review. Adv Space Res 3: $153-158$

8. Hammond TG, Hammond JM (2001) Optimized suspension culture: the rotating-wall vessel. Am J Physiol Renal Physiol 281: F12-F25.

9. Todd P (1989) Gravity-dependent phenomena at the scale of the single cell. ASGSB Bull 2: 95-113.

10. Klein-Nulend J, Bacabac RG, Veldhuijzen JP, van Loon JJWA (2003) Microgravity and bone cell mechanosensitivity. Adv Space Res 32: 1551-1559.

11. Pardo SJ, Patel MJ, Sykes MC, Platt MO, Boyd NL, et al. (2005) Simulated microgravity using the Random Positioning Machine inhibits differentiation and alters gene expression profiles of 2T3 preosteoblasts. Am J Physiol Cell Physiol 288 : C1211-C1221. [Crossref]

12. Monici M, Fusi F, Paglierani M, Marziliano N, Cogoli A, et al. (2006) Modeled gravitational unloading triggers differentiation and apoptosis in preosteoclastic cells. J Cell Biochem 98: 65-80.

13. Nabavi N, Khandani A, Camirand A, Harrison RE (2011) Effects of microgravity on osteoclast bone resorption and osteoblast cytoskeletal organization and adhesion. Bone 49: 965-974. [Crossref]

14. Ohshima H (2006) Bone loss and bone metabolism in astronauts during long-duration space flight. Clin Calcium 16: 81-85.

15. Tamma R, Colaianni G, Camerino C, Di Benedetto A, Greco G, et al. (2009) Microgravity during spaceflight directly affects in vitro osteoclastogenesis and bone resorption. FASEB J 23: 2549-2554.

16. Fitts RH, Riley DR, Widrick JJ (2001) Functional and structural adaptations of skeletal muscle to microgravity. J Exp Biol 204: 3201-3208.

17. Fitts RH, Trappe SW, Costill DL, Gallagher PM, Creer AC, et al. (2010) Prolonged space flight-induced alterations in the structure and function of human skeletal muscle fibres. J Physiol 588: 3567-3592.

18. Aubert AE, Beckers F, Verheyden B (2005) Cardiovascular function and basics of physiology in microgravity. Acta Cardiol 60: 129-151.

19. Convertino VA (2005) Consequences of cardiovascular adaptation to spaceflight: implications for the use of pharmacological countermeasures. Gravit Space Biol Bull 18: 59-69. [Crossref]

20. Durnova GN, Burkovskaia TE, Vorotnikova EV, Kaplanskiǐ AS, Arustamov OV (1991) The Effect of Weightlessness on Fracture Healing of Rats Flown on the Biosatellite Cosmos-2044. Kosm Biol Aviakosm Med 25: 29-33.

21. Kirchen ME, O’Connor KM, Gruber HE, Sweeney JR, Fras IA, et al. (1995) Effects of Microgravity on Bone Healing in a Rat Fibular Osteotomy Model. Clin Orthop Relat Res 231-242. [Crossref]

22. Radek KA, Baer LA, Eckhardt J, DiPietro LA, Wade CE (2008) Mechanical unloading impairs keratinocyte migration and angiogenesis during cutaneous wound healing. $J$ Appl Physiol (1985) 104: 1295-1303.

23. Sonnenfeld G (2005) The immune system in space, including earth-based benefits of space-based research. Curr Pharm Biotechnol 6: 343-349.

24. Stowe RP, Sams CF, Pierson DL (2011) Adrenocortical and immune responses following short- and long duration spaceflight. Aviat Space Environ Med 82: 627-634.

25. Blaber E, Marçal H, Burns BP (2010) Bioastronautics: the influence of microgravity on astronaut health. Astrobiology 10: 463-473.

26. Donahue TLH, Haut TR, Yellowley CE, Donahue HJ, Jacobs CR (2003) Mechanosensitivity of bone cells to oscillating fluid flow induced shear stress may be modulated by chemotransport. J Biomech 36: 1363-1371.

27. Collet P, Uebelhart D, Vico L, Moro L, Hartmann D, et al. (1997) Effects of 1- and 6-month spaceflight on bone mass and biochemistry in two humans. Bone 20: 547-551. [Crossref]

28. Marie PJ, Jones D, Vico L, Zallone A, Hinsenkamp M, et al. (2000) Osteobiology, strain, and microgravity: part I. Studies at the cellular level. Calcif Tissue Int 67: 2-9. 
29. Robling AG, Burr DB, Turner CH (2001) Recovery periods restore mechanosensitivity to dynamically loaded bone. J Exp Biol 204: 3389-3399.

30. Sessions ND, Halloran BP, Bikle DD, Wronski TJ, Cone CM, et al. (1989) Bone response to normal weight bearing after a period of skeletal unloading. Am J Physiol Endocrinol Metab 257: E606-E610. [Crossref]

31. Siddiqui JA, Partridge NC (2016) Physiological bone remodeling: Systematic regulation and growth factor involvement. Physiology (Bethesda) 31: 233-245.

32. Sims NA, John Martin T (2014) Coupling the activities of bone formation and resorption: a multitude of signals within the basic multicellular unit. Bonekey Rep 3: 481. [Crossref]

33. Xiao W, Li S, Pacios S, Wang Y, Graves DT (2016) Bone remodeling under pathological conditions. Front Oral Biol 18: 17-27.

34. Caillot-Augusseau A, Lafage-Proust MH, Soler C, Pernod J, Dubois F, et al. (1998) Bone formation and resorption biological markers in cosmonauts during and after a 180-day space flight (Euromir 95). Clin Chem 44: 578-585.

35. Carmeliet G, Bouillon R (1999) The effect of microgravity on morphology and gene expression of osteoblasts in vitro. FASEB J 13: S129-S134.

36. Colleran PN, Wilkerson MK, Bloomfield SA, Suva LJ, Turner RT, et al. (2000) Alterations in skeletal perfusion with simulated microgravity: a possible mechanism for bone remodeling. J Appl Physiol 89: 1046-1054. [Crossref]

37. Bucaro MA, Zahm AM, Risbud MV, Ayyaswamy PS, Mukundakrishnan K, et al. (2007) The effect of simulated microgravity on osteoblasts is independent of the induction of apoptosis. J Cell Biochem 102: 483-495.

38. Pittenger MF, Mackay AM, Beck SC, Jaiswal RK, Douglas R, et al. (1999) Multilineage potential of adult human mesenchymal stem cells. Science 284: 143-147.

39. Yuge L, Sasaki A, Kawahara Y, Wu SL, Matsumoto M, et al. (2011) Simulated microgravity maintains the undifferentiated state and enhances the neural repair potential of bone marrow stromal cells. Stem Cells Dev 20: 893-900.

40. Ozcivici E, Luu YK, Adler B, Qin YX, Rubin J, et al. (2010) Mechanical signals as anabolic agents in bone. Nat Rev Rheumatol 6: 50-59. [Crossref]

41. Zayzafoon M, Gathings WE, McDonald JM (2004) Modeled microgravity inhibits osteogenic differentiation of human mesenchymal stem cells and increases adipogenesis. Endocrinology 145: 2421-2432.

42. Huang Y, Dai ZQ, Ling SK, Zhang HY, Wan YM, et al. (2009) Gravity, a regulation factor in the differentiation of rat bone marrow mesenchymal stem cells. J Biomed Sci 16: 87. [Crossref]

43. Meng R, Xu HY, Di SM, Shi DY, Qian AR, et al. (2011) Human mesenchymal stem cells are sensitive to abnormal gravity and exhibit classic apoptotic features. Acto Biochim Biophys Sin 43: 133-142.

44. Yang RS, Lin WL, Chen YZ, Tang CH, Huang TH, et al. (2005) Regulation by ultrasound treatment on the integrin expression and differentiation of osteoblasts. Bone 36: 276-283. [Crossref]

45. Hsu HC, Fong YC, Chang CS, Hsu CJ, Hsu SF, et al. (2007) Ultrasound induces cyclooxygenase-2 expression through integrin, integrin-linked kinase, Akt, NF-kappaB and p300 pathway in human chondrocytes. Cell Signal 19: 2317-2328.

46. Grafe I, Alexander S, Peterson JR, Snider TN, Levi B, et al. (2018) TGF- $\beta$ Family Signaling in Mesenchymal Differentiation. Cold Spring Harb Perspect Biol 10: a022202.

47. Chen D, Gong Y, Xu L, Zhou M, Li J, et al. (2019) Bidirectional regulation of osteogenic differentiation by the FOXO subfamily of Forkhead transcription factors in mammalian MSCs. Cell Prolif 52: e125440.

48. Papachroni KK, Karatzas DN, Papavassiliou KA, Basdra EK, Papavassiliou AG (2009) Mechanotransduction in osteoblast regulation and bone disease. Trends Mol Med 15: 208-216. [Crossref]

49. Smith SM, Wastney ME, O’Brien KO, Morukov BV, Larina IM, et al. (2005) Bone markers, calcium metabolism, and calcium kinetics during extended-duration space flight on the mir space station. J Bone Miner Res 20: 208-218.

50. Carmeliet G, Nys G, Bouillon R (1997) Microgravity reduces the differentiation of human osteoblastic MG-63 cells. J Bone Miner Res 12: 786-794. [Crossref]

51. Pan Z, Yang J, Guo C, Shi D, Shen D, et al. (2008) Effects of hindlimb unloading on ex vivo growth and osteogenic/adipogenic potentials of bone marrow-derived mesenchymal stem cells in rats. Stem Cells Dev 17: 795-804.
52. Arfat Y, Xiao WZ, Iftikhar S, Zhao F, Li DJ, et al. (2014) Physiological effects of microgravity on bone cells. Calcif Tissue Int 94: 569-579.

53. Dai Z, Wu F, Chen J, Xu H, Wang H, et al. (2013) Actin microfilament mediates osteoblast Cbfa1 responsiveness to BMP2 under simulated microgravity. PLoS One 8: e63661. [Crossref]

54. Kumei Y, Shimokawa H, Ohya K, Katano H, Akiyama H, et al. (2007) Small GTPase Ras and Rho expression in rat osteoblasts during spaceflight. Ann N Y Acad Sci 1095 292-299.

55. Guignandon A, Lafage-Proust MH, Usson Y, Laroche N, Caillot-Augusseau A, et al (2001) Cell cycling determines integrin-mediated adhesion in osteoblastic ROS 17/2.8 cells exposed to space-related conditions. FASEB $J$ 15: 2036-2038. [Crossref]

56. Starr DA (2007) Communication between the cytoskeleton and the nuclear envelope to position the nucleus. Mol Biosyst 3: 583-589.

57. Qian AR, Li D, Han J, Gao X, Di SM, et al. (2012) Fractal dimension as a measure of altered actin cytoskeleton in MC3T3-E1 cells under simulated microgravity using 3-D/2-D clinostats. IEEE Trans Biomed Eng 59: 1374-1380.

58. Kumei Y, Morita S, Katano H, Akiyama H, Hirano M, et al. (2006) Microgravity signa ensnarls cell adhesion, cytoskeleton, and matrix proteins of rat osteoblasts: osteopontin, CD44, osteonectin, and alpha-tubulin. Ann N Y Acad Sci 1090: 311-317.

59. Higashibata A, Imamizo-Sato M, Seki M, Yamazaki T, Ishioka N (2006) Influence of simulated microgravity on the activation of the small GTPase Rho involved in cytoskeletal formation--molecular cloning and sequencing of bovine leukemiaassociated guanine nucleotide exchange factor. BMC Biochem 7: 19.

60. Kondo H, Yumoto K, Alwood JS, Mojarrab R, Wang A, et al. (2010) Oxidative stress and gamma radiation-induced cancellous bone loss with musculoskeletal disuse. $J \mathrm{Appl}$ Physiol (1985) 108: 152-161. [Crossref]

61. Saxena R, Pan G, Dohm ED, McDonald JM (2011) Modeled microgravity and hindlimb unloading sensitize osteoclast precursors to RANKL-mediated osteoclastogenesis. $J$ Bone Miner Metab 29: 111-122. [Crossref]

62. Rucci N, Rufo A, Alamanou M, Teti A (2007) Modeled microgravity stimulates osteoclastogenesis and bone resorption by increasing osteoblast RANKL/OPG ratio. J Cell Biochem 100: 464-473.

63. Segovia-Silvestre T, Neutzsky-Wulff AV, Sorensen MG, Christiansen C, Bollerslev J, et al. (2009) Advances in osteoclast biology resulting from the study of osteopetrotic mutations. Hum Genet 124: 561-577. [Crossref]

64. Tamma R, Colaianni G, Camerino C, Di Benedetto A, Greco G, et al. (2009) Microgravity during spaceflight directly affects in vitro osteoclastogenesis and bone resorption. FASEB J 23: 2549-2554.

65. Sambandam Y, Blanchard JJ, Daughtridge G, Kolb RJ, Shanmugarajan S, et al. (2010) Microarray profile of gene expression during osteoclast differentiation in modelled microgravity. J Cell Biochem 111: 1179-1187.

66. Tanaka K, Nishimura N, Kawai Y (2017) Adaptation to microgravity, deconditioning, and countermeasures. J Physiol Sci 67: 271-281.

67. Narici MV, de Boer MD (2011) Disuse of the musculo-skeletal system in space and on earth. Eur J Appl Physiol 111: 403-420.

68. Convertino VA (1990) Physiological adaptations to weightlessness: effects on exercise and work performance. Exerc Sport Sci Rev 18: 119-166. [Crossref]

69. di Prampero PE, Narici MV (2003) Muscles in microgravity: from fibres to human motion. J Biomech 36: 403-412.

70. Gardetto PR, Schluter JM, Fitts RH (1989) Contractile function of single muscle fibers after hindlimb suspension. J Appl Physiol (1985) 66: 2739-2749.

71. Ohira Y, Jiang B, Roy RR, Oganov V, Ilyina-Kakueva E, et al. (1992) Rat soleus muscle fiber responses to 14 days of spaceflight and hindlimb suspension. J Appl Physiol (1985) 73: 51S-57S. [Crossref]

72. Roy RR, Bello MA, Bouissou P, Edgerton VR (1987) Size and metabolic properties of fibers in rat fast-twitch muscles after hindlimb suspension. $J$ Appl Physiol 62: 23482357.

73. Fitts RH, Riley DR, Widrick JJ (2000) Physiology of a microgravity environment invited review: microgravity and skeletal muscle. J Appl Physiol 89: 823-839.

74. Slentz DH, Truskey GA, Kraus WE (2001) Effects of chronic exposure to simulated microgravity on skeletal muscle cell proliferation and differentiation. In Vitro Cell Dev Biol Anim 37: 148-156. 
75. Kalb R, Solomon D (2007) Space exploration, Mars, and the nervous system. Arch Neurol 64: 485-490. [Crossref]

76. LeBlanc A, Rowe R, Schneider V, Evans H, Hedrick T (1995) Regional muscle loss after short duration spaceflight. Aviat Space Environ Med 66: 1151-1154.

77. Akima H, Kawakami Y, Kubo K, Sekiguchi C, Ohshima H, et al. (2000) Effect of short-duration spaceflight on thigh and leg muscle volume. Med Sci Sports Exerc 32: 1743-1747.

78. Widrick JJ, Knuth ST, Norenberg KM, Romatowski JG, Bain JL, et al. (1999) Effect of a 17 days spaceflight on contractile properties of human soleus muscle fibers. J Physiol 516: 915-930. [Crossref]

79. Edgerton VR, Zhou MY, Ohira Y, Klitgaard H, Jiang B, et al. (1995) Human fiber size and enzymatic properties after 5 and 11 days of spaceflight. J Appl Physiol (1985) 78: 1733-1739.

80. Tarantino U, Baldi J, Celi M, Rao C, Liuni FM, et al. (2013) Osteoporosis and sarcopenia: the connections. Aging Clin Exp Res 25: S93-S95. [Crossref]

81. Avin KG, Bloomfield SA, Gross TS, Warden SJ (2015) Biomechanical aspects of the muscle-bone interaction. Curr Osteoporos Rep 13:1-8.

82. Li GB, Zhang L, Wang DE, AlQudsy L, Jiang JX, et al. (2019) Muscle-bone crosstalk and potential therapies for sarco-osteoporosis. J Cell Biochem 120: 14262-14273.

83. Brotto M, Boneyard L (2015) Bone and muscle: Interactions beyond mechanical. Bone 80: 109-114.

84. Paintin J, Cooper C, Dennison E (2018) Osteosarcopenia. Br J Hosp Med (Lond) 79: 253-258.

85. Edwards MH, Dennison EM, Sayer AA, Fielding R, Cooper C (2015) Osteoporosis and sarcopenia in older age. Bone 80: 126-130.

86. Fuggle N, Shaw S, Dennison E, Cooper C (2017) Sarcopenia. Best Pract Res Clin Rheumatol 31: 218-242.

87. Di Girolamo DJ, Kiel DP, Esser KA (2013) Bone and skeletal muscle: neighbors with close ties. J Bone Miner Res 28: 1509-1518.

88. Sartori R, Sandri M (2015) BMPs and the muscle-bone connection. Bone 80: 37-42. [Crossref]

89. Sartori R, Schirwis E, Blaauw B, Bortolanza S, Zhao J, et al. (2013) BMP signaling controls muscle mass. Nat Genet 45: 1309-1318.

90. Salazar VS, Gamer LW, Rosen V (2016) BMP signalling in skeletal development, disease and repair. Nat Rev Endocrinol 12: 203-221.

91. Brazil DP, Church RH, Surae S, Godson C, Martin F (2015) BMP signalling: agony and antagony in the family. Trends Cell Biol 25: 249-264. [Crossref]
92. Tarantino U, Scimeca M, Piccirilli E, Tancredi V, Baldi J, et al. (2015) Sarcopenia: a histological and immunohistochemical study on age-related muscle impairment. Aging Clin Exp Res 27: S51-S60.

93. Scimeca M, Bonanno E, Piccirilli E, Baldi J, Mauriello A, et al. (2015) Satellite Cells CD44 Positive Drive Muscle Regeneration in Osteoarthritis Patients. Stem Cells Int 2015: 469459.

94. Tarantino U, Baldi J, Scimeca M, Piccirilli E, Piccioli A, et al. (2016) The role of sarcopenia with and without fracture. Injury 47: S3-S10.

95. Yin H, Price F, Rudnicki MA (2013) Satellite cells and the muscle stem cell niche Physiol Rev 93: 23-67.

96. Scimeca M, Piccirilli E, Mastrangeli F, Rao C, Feola M, et al. (2017) Bone Morphogenetic Proteins and myostatin pathways: key mediator of human sarcopenia. J Transl Med 15: 34. [Crossref]

97. Thomas M, Langley B, Berry C, Sharma M, Kirk S, et al. (2000) Myostatin, a negative regulator of muscle growth, functions by inhibiting myoblast proliferation. $J$ Biol Chem 275: 40235-40243.

98. McPherron AC, Lee SJ (2002) Suppression of body fat accumulation in myostatindeficient mice. J Clin Invest 109: 595-601.

99. Tarantino U, Cariati I, Marini M, D'Arcangelo G, Tancredi V, et al. (2020) Effects of Simulated Microgravity on Muscle Stem Cells Activity. Cell Physiol Biochem 54: 736-747. [Crossref]

100. Bloomfield SA (1997) Changes in musculoskeletal structure and function with prolonged bed rest. Med Sci Sports Exerc 29: 197-206.

101. Reilly B, Franklin C (2016) Prevention of muscle wasting and osteoporosis: the value of examining novel animal models. J Exp Biol 219: 2582-2595.

102. McCarthy EF (2011) Perspective: skeletal complications of space flight. Skeletal Radiol 40: 661-663.

103. Lang T, LeBlanc A, Evans H, Lu Y, Genant H, et al. (2004) Cortical and trabecular bone mineral loss from the spine and hip in long-duration spaceflight. J Bone Miner Res 19: 1006-1012. [Crossref]

104. Lang TF, Leblanc AD, Evans HJ, Lu Y (2006) Adaptation of the proximal femur to skeletal reloading after long-duration spaceflight. J Bone Miner Res 21: 1224-1230.

105. Burr DB (1997) Muscle strength, bone mass, and age-related bone loss. $J$ Bone Miner Res 12: 1547-1551.

106. Lloyd SA, Lang CH, Zhang Y, Paul EM, Laufenberg LJ, et al. (2014) Interdependence of muscle atrophy and bone loss induced by mechanical unloading. J Bone Miner Res 29: 1118-1130. [Crossref]

107. Keyak JH, Koyama AK, LeBlanc A, Lu Y, Lang TF, et al. (2009) Reduction in proximal femoral strength due to long-duration spaceflight. Bone 44: 449-453.

Copyright: $\odot 2020$ Tarantino U. This is an open-access article distributed under the terms of the Creative Commons Attribution License, which permits unrestricted use, distribution, and reproduction in any medium, provided the original author and source are credited. 